Check for updates

Cite this: RSC Adv., 2019, 9, 18868

Received 8th April 2019

Accepted 27th May 2019

DOI: 10.1039/c9ra02618e

rsc.li/rsc-advances

\title{
A novel synthesis of silver nanowires by using 6- chlorohexylzinc bromide as an additive for low haze transparent conductive films
}

\author{
Ximin Yuan, Hongwei Yang, iD * Yuxiu Li, Yunxiu Chao, Yao Li, Li Chen \\ and Jialin Chen*
}

\begin{abstract}
High-quality silver nanowires (AgNWs) with a small diameter of $\sim 20 \mathrm{~nm}$ and a length of $\sim 40 \mu \mathrm{m}$ were prepared by using a novel organic 6-chlorohexylzinc bromide as an assistant additive. The diameter of as-synthesized AgNWs was confirmed to be strongly dependent on the dosage of 6-chlorohexylzinc bromide. Moreover, a two-dimensional (2D) transparent conductive film (TCF) with an excellent optical performance was fabricated by as-synthesized AgNWs, which has a $90.3 \%$ transmittance and low haze value of $<1.0 \%$ at a sheet resistance of $48.7 \Omega \mathrm{sq}^{-1}$.
\end{abstract}

\section{Introduction}

Currently, indium tin oxide (ITO), which is a transparent conductive material, widely used in highly transparent display devices, because it has relatively low resistivity. However, the ITO films are brittle and easily broken by an externally applied bending force, which have high brittleness and poor flexibility. ${ }^{\mathbf{1 , 2}}$ With the development of technology and society, in order to meet people's needs for medicine, ${ }^{3}$ flexible touch screens, ${ }^{4}$ solar cells, ${ }^{5}$ wearable devices, ${ }^{6}$ etc., AgNWs are attracting much attention for their unique electronic conductivity and good flexibility. Therefore, it is very necessary to study and prepare high-quality AgNWs, making it become an ITO replacement material with realistic application prospects, especially as a popular material for flexible screen production.

Thus far, there are many ways to prepare AgNWs, for example, electrochemical method, ${ }^{7}$ hydrothermal method, ${ }^{8}$ and microwave-assisted method, ${ }^{9}$ etc. Xi et al. successfully synthesized AgNWs with different diameters $(28 \mathrm{~nm}, 38 \mathrm{~nm}, 55 \mathrm{~nm}, 80$ $\mathrm{nm})$ through direct-current electrochemical deposition technique, but needed to prepare the template in advance, and it also required complex post-processing. ${ }^{7} \mathrm{Xu}$ et al. synthesized AgNWs with $30 \mathrm{~nm}$ in diameter by hydrothermal process in the presence of gemini surfactant; however, the operation was complex and needed two surfactants. ${ }^{8}$ Ying et al. synthesized AgNWs with the diameter of $40 \mathrm{~nm}$ through a microwaveassisted polyol reduction method using 1,2-ethanedithiol as both a reducing reagent and a solvent. But the length of AgNWs was as low as $\sim 2.2 \mu \mathrm{m} .{ }^{9}$ In contrast, AgNWs are mostly prepared by the polyol method. For example, You et al. prepared the

State Key Laboratory of Advanced Technologies for Comprehensive Utilization of Platinum Metals, Kunming Institute of Precious Metals, 650106 Kunming, People's Republic of China. E-mail: nanolab@ipm.com.cn
AgNWs with diameter of $20 \mathrm{~nm}$ by reducing silver nitrate in ethanol solution with dodecylamine as a complex. But the reaction time was too long (heating at $100{ }^{\circ} \mathrm{C}$ for $24 \mathrm{~h}$ ). ${ }^{10}$ Also, Silva et al. synthesized AgNWs with the diameter of $20 \mathrm{~nm}$ by using sodium bromide as a capping agent. However, the length of AgNWs synthesized only approached $20 \mu \mathrm{m} .{ }^{\mathbf{1 1}}$ Lee et al. successfully prepared $15 \mathrm{~nm}$ AgNWs, which required two additives ( $\mathrm{NaCl}$ and $\mathrm{KBr}$ ) and high pressure conditions. ${ }^{12}$ Thus, it is still necessary to develop a facile method for synthesis of high-quality AgNWs.

In this study, organic 6-chlorohexylzinc bromide was first used to replace the traditional inorganic halide to prepare highquality AgNWs. The probable controlled mechanism of 6chlorohexylzinc bromide on the formation of AgNWs was discussed in details. Moreover, flexible transparent conductive films with low haze and resistance were thus fabricated based on the as-prepared AgNWs, indicating the potential application in flexible screen.

\section{Experimental}

\subsection{Materials}

Silver nitrate $\left(\mathrm{AgNO}_{3}, \geq 99.8 \%\right)$ was purchased from Sinopharm Chemical Reagent Co., Ltd. Ethylene glycol (EG, $\geq 99.0 \%$ ), polyvinylpyrrolidone (PVP, $M_{\mathrm{w}} \sim 1300000$ ) were acquired from Sigma Aldrich. Ethanol $\left(\mathrm{C}_{2} \mathrm{H}_{5} \mathrm{OH}, \geq 99.0 \%\right)$ was acquired from Xilong Chemical Co., Ltd. 6-Chlorohexylzinc bromide (6CHZB, $0.5 \mathrm{M}$ in tetrahydrofuran, density of 0.89 ) was purchased from Fluorochem Ltd., and hydroxyethyl cellulose (HEC, QP-100 MH) was purchased from Dow. Deionized (DI) water was prepared by using a laboratory water purification system with a resistivity of not less than $18.2 \mathrm{M} \Omega \mathrm{cm}$ and an operating temperature of $25{ }^{\circ} \mathrm{C}$. All chemicals were used as received without further purification. 


\subsection{Methods}

2.2.1. Synthesis of high-quality AgNWs. High-quality AgNWs were synthesized by the 6CHZB-assisted polyol method. First, three solutions were prepared: (a) $1.263 \mathrm{~g}$ PVP was dissolved in $30 \mathrm{~mL}$ EG, (b) $1.122 \mathrm{~g} \mathrm{AgNO}_{3}$ was dissolved in $20 \mathrm{~mL}$ EG, (c) appropriate amount of 6CHZB was dissolved in $11 \mathrm{~mL}$ EG. Second, (a), (c) and $239 \mathrm{~mL}$ EG were added to flask at $25{ }^{\circ} \mathrm{C}$ and stirred for $30 \mathrm{~min}$. Next, (b) was added to the above solution and continued stirring for $10 \mathrm{~min}$. The solution was then allowed to stand at $25{ }^{\circ} \mathrm{C}$ for $2 \mathrm{~h}$. Subsequently, the solution was added to $500 \mathrm{~mL}$ round bottom flask and heated at a fixed temperature for $20 \mathrm{~min}$. The final solution was naturally cooled to room temperature and then centrifuged using ethanol and DI water several times to remove the EG, PVP and other impurities. After the final centrifugation, the AgNWs were redispersed into DI water for further use.

2.2.2. Preparation of AgNWs ink. An AgNWs ink was prepared by using HEC as the resinous material. First, $1 \mathrm{wt} \%$ HEC aqueous solution was produced by dissolving $1 \mathrm{~g}$ HEC in $100 \mathrm{~mL}$ of DI water. Second, appropriate amount of AgNWs were re-dispersed into $1 \mathrm{wt} \%$ HEC aqueous solution (the final ratio of AgNWs to HEC is $2: 1$ ) by stirring at $25^{\circ} \mathrm{C}$ for $3 \mathrm{~h}$, and eventually the uniform AgNWs ink was obtained.

2.2.3. Fabrication of AgNWs TCFs. AgNWs TCFs were manufactured by a bar coating process. In the first, AgNWs/HEC solution was precisely added to the polyethylene terephthalate (PET) substrate. Next, a uniform mixture was applied to the PET substrate by using an automatic film applicator (BEVS1811/2) at a rod speed of $50 \mathrm{~mm} \mathrm{~s}^{-1}$ with a rod diameter of $3 \mu \mathrm{m}$, and then cured at $130{ }^{\circ} \mathrm{C}$ for $10 \mathrm{~min}$, finally forming a two-dimensional AgNWs network on the PET substrate.

\subsection{Characterization}

Phase identification was performed using X-ray diffraction (XRD) of copper $\mathrm{K} \alpha$ radiation $(\lambda=1.54056 \AA$ ), where the intensities of the diffracted X-rays were recorded as a function of $2 \theta$ (scanning in steps of $0.02^{\circ} \mathrm{s}^{-1}$ from $20^{\circ}$ to $90^{\circ}$ ). The chemical composition characterization was performed by X-ray photoemission spectroscopy (XPS) with a PHI5000 VersaprobeII instrument using monochromatic aluminum $\mathrm{K} \alpha \mathrm{X}$-radiation, which was operated in the constant-pass energy mode. The binding energy scale was calibrated by measuring the C1s peak at $284.8 \mathrm{eV}$. The microstructures of the products were observed on a FEI-Versa 3D field emission scanning electron microscopy (FE-SEM) operating at $30 \mathrm{kV}$. High-resolution transmission electron microscopy (HRTEM) was examined on a Philips CM 200-FEG microscope (200 kV, $\left.C_{\mathrm{s}}=1.35 \mathrm{~mm}\right)$, and the transmission electron micrography (TEM, Tecnai G2-TF30) was performed on device at a stimulation voltage of $120 \mathrm{kV}$. The UV-vis spectra with a scan range from $190 \mathrm{~nm}$ to $900 \mathrm{~nm}$ of the sample were performed on a UV-vis spectrophotometer (PERSEE Genera TU-1901). The photoelectric properties of transparent conductive films were studied by SGW-820 transmittance and haze analyzer. The sheet resistance of the AgNW-based TCF was examined using a four-point probe (SB 100A/2) with a probe current of $2 \mathrm{~mA}$.

\section{Results and discussion}

The FE-SEM images of AgNWs prepared under different molar ratio of $6 \mathrm{CHZB} / \mathrm{AgNO}_{3}$ are shown in Fig. 1 . It can be clearly seen from the FE-SEM images that when the molar ratio of $6 \mathrm{CHZB} / \mathrm{AgNO}_{3}$ was $0.01,0.02,0.03$, and 0.04 , the corresponding finest diameter of AgNWs was $31 \mathrm{~nm}, 22 \mathrm{~nm}$, $29 \mathrm{~nm}$, and $33 \mathrm{~nm}$, respectively. It was apparent that AgNWs have the finest diameter when the molar ratio of $6 \mathrm{CHZB} /$ $\mathrm{AgNO}_{3}$ was 0.02 . Therefore, 0.02 was determined as the optimum molar ratio of $6 \mathrm{CHZB} / \mathrm{AgNO}_{3}$.

The effect of reaction temperature on the morphology of AgNWs were further studied, the control experiments at different temperatures were carried out, and the corresponding FE-SEM images of AgNWs preparation were displayed in Fig. 2. When the reaction temperature was $160^{\circ} \mathrm{C}$, the diameter of the finest AgNW was $36 \mathrm{~nm}$ (Fig. 2(a)). As increasing the reaction temperature up to $165{ }^{\circ} \mathrm{C}$, the finest diameter of $\mathrm{AgNW}$ was $30 \mathrm{~nm}$ (Fig. 2(b)). When the reaction temperature was $175^{\circ} \mathrm{C}$, it is clear that the finest diameter of AgNW could reach $26 \mathrm{~nm}$ (Fig. 2(d)). By contrast, when the reaction temperature was $170{ }^{\circ} \mathrm{C}$, the $\mathrm{AgNWs}$ have the finest diameter of $22 \mathrm{~nm}$ (Fig. 2(c)). Based on the above discussion, 0.02 was determined as the optimum molar ratio of $6 \mathrm{CHZB} /$ $\mathrm{AgNO}_{3}$ and the optimal reaction temperature was $170{ }^{\circ} \mathrm{C}$. The following discussion below is thereby under these conditions.

The XRD pattern of the as-synthesized AgNWs is depicted in Fig. 3. The apparent five peaks at $38^{\circ}, 44^{\circ}, 64^{\circ}, 77^{\circ}$, and $81^{\circ}$ corresponded to (111), (200), (220), (311), and (222) Bragg reflections of $\mathrm{Ag}$, respectively. By comparing the curve with JCPDS (No. 04-0783) of Ag, it can be fully indexed to a pure phase of face-centered cubic (fcc) Ag crystal ( $a=b=c=4.086 \AA$, space group $F m \overline{3} m$ (225)). In addition, no traces of other compounds could be detected, indicating the high purity, which is in good agreement with previous studies. ${ }^{\mathbf{1 3 , 1 4}}$ It is worth noting that the intensity of (111) peak is relatively high, indicating that the AgNWs grew along the (111) Bragg reflection.

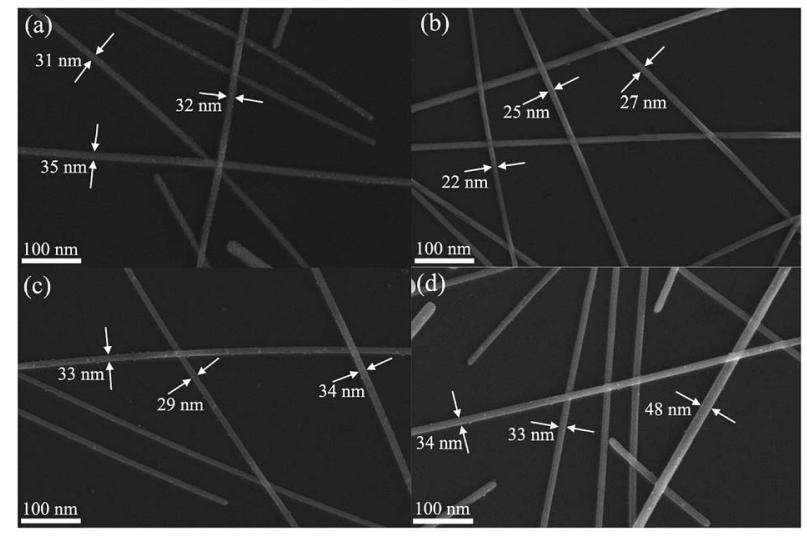

Fig. 1 FE-SEM images of AgNWs prepared under different molar ratio of $6 \mathrm{CHZB} / \mathrm{AgNO}_{3}$ : (a) 0.01 , (b) 0.02 , (c) 0.03 , and (d) 0.04 , respectively, reaction temperature $=170{ }^{\circ} \mathrm{C}$. 


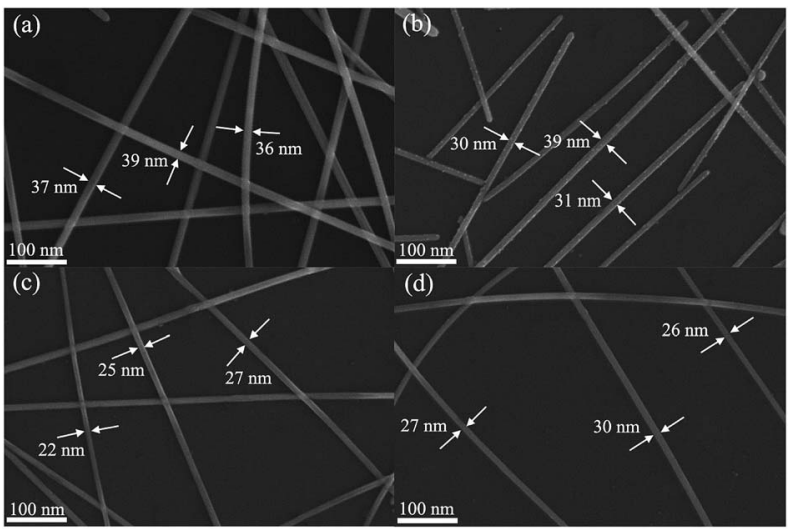

Fig. 2 FE-SEM images of AgNWs prepared at different reaction temperatures: (a) $160{ }^{\circ} \mathrm{C}$, (b) $165^{\circ} \mathrm{C}$, (c) $170{ }^{\circ} \mathrm{C}$, and (d) $175{ }^{\circ} \mathrm{C}$, respectively, molar ratio of $6 \mathrm{CHZB} / \mathrm{AgNO}_{3}=0.02$.

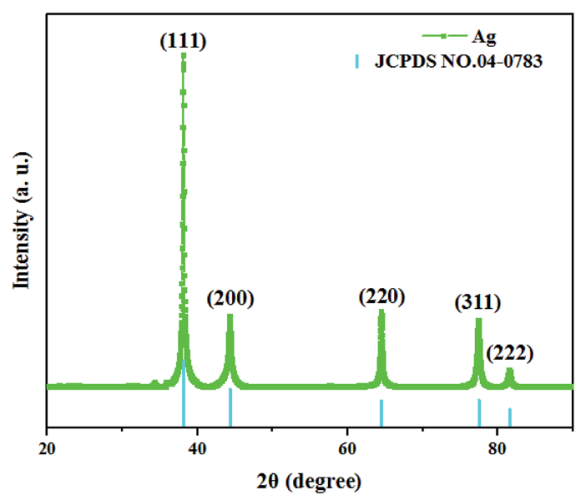

Fig. 3 XRD pattern of the as-synthesized AgNWs.

To better understand the chemical composition of the assynthesized AgNWs, XPS analysis was further carried out, and the results were shown in Fig. 4. Fig. 4(a) is a full-spectrum scan of the as-synthesized AgNWs, which can be clearly found that $\mathrm{Ag}$ is the only elements detected except the elements $(\mathrm{C}, \mathrm{O})$ from reference substance. The high resolution Ag $3 \mathrm{~d}$ XPS spectrum shows two prominent peaks around 367 and $373 \mathrm{eV}$, respectively in good agreement with silver metallic values. ${ }^{15}$

The microscopic morphology of the as-synthesized AgNWs was observed by FE-SEM, and the images of AgNWs at different magnifications are shown in Fig. 5. Fig. 5(a)-(c) clearly shows the structural features of AgNW with a thin and uniform diameter and a narrow size distribution. According to the enlarged FE-SEM image (Fig. 5(a)), the diameter of AgNWs varied from 22 to $27 \mathrm{~nm}$ with a narrow size distribution. In addition, one can see that the surface was smooth without tiny structures. As can be seen from Fig. 5(d), the length of the as-synthesized AgNW was estimated to be 35 to $40 \mu \mathrm{m}$. More importantly, no other $\mathrm{Ag}$ nanostructures such as polyhedrons, spheres and cubes were observed, demonstrating the high uniformity of AgNWs.
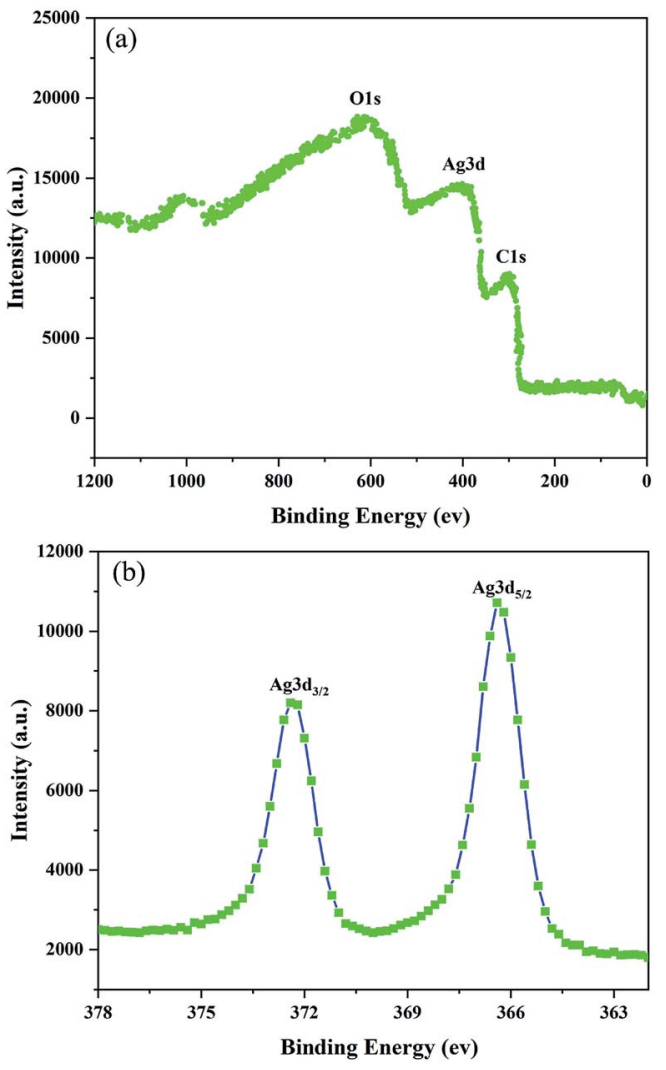

Fig. 4 XPS spectra of the as-synthesized AgNWs.

The as-synthesized AgNWs were further characterized by FETEM and selected area electron diffraction (SAED) analyses, as shown in Fig. 6. Obviously, the ultra-fine AgNWs have been successfully synthesized. As is clear from Fig. 6(b), AgNW was divided into two halves by a double plane parallel with longitudinal axis, and the insetted SAED pattern further proves that AgNWs has a twin crystalline structure. Further, well-developed lattice fringes having a regular pitch of $0.14 \mathrm{~nm}$ can be observed, which was consistent with the separation of Ag (220) crystal plane, and the high crystallinity of the synthesized AgNW was confirmed together with the above XRD analysis.

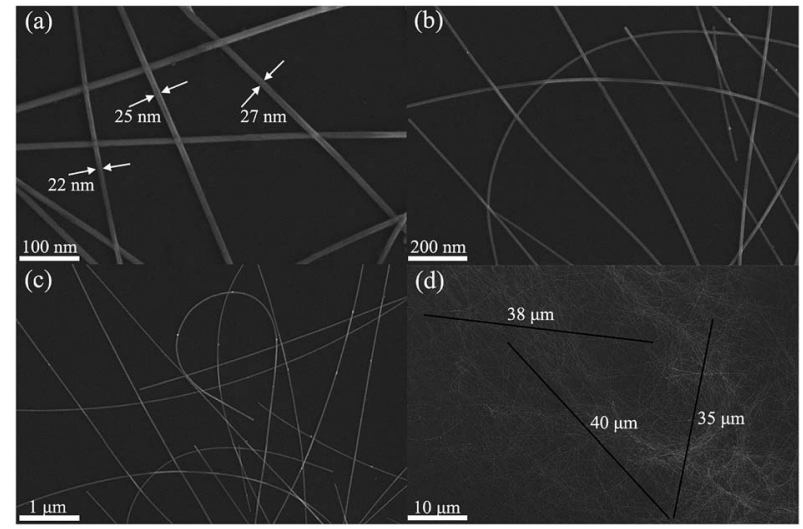

Fig. 5 FE-SEM images of AgNWs at different magnifications. 


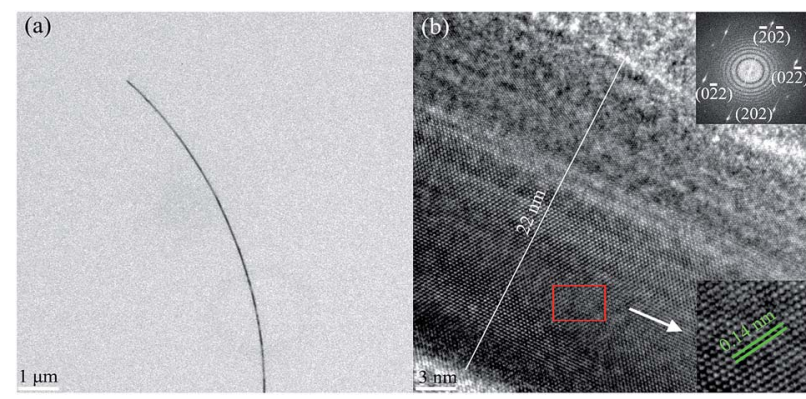

Fig. 6 FE-TEM images and SAED pattern (inset) of the as-synthesized AgNWs.

To investigate the possible formation mechanism of AgNWs in this work, samples produced at different stages were obtained and the corresponding FE-SEM results were shown in Fig. 7. Firstly, as can be seen from Fig. 7(a), after standing at room temperature for $2 \mathrm{~h}$, almost no substance was visible, suggesting that the reaction did not occur obviously during this process. Second, the silver ions should be reduced to silver atoms on the $\mathrm{AgBr}$ particles through EG as shown in Fig. 7(b). In Fig. 7(c), heating was continued and small silver decahedral seeds were formed subsequently on the surface of $\mathrm{AgBr}$ particles. When the solution was further heated to 15 min (Fig. 7(d)), the one-dimensional growth of $\mathrm{Ag}$ decahedral seeds eventually leads to the formation of AgNWs. Finally, the thick AgNWs continued to grow and produced the ultra-fine AgNWs (Fig. 7(e)).

Everyone knows that nano-metals, especially silver, exhibit excessive optical phenomena directly related to surface plasmon resonance (SPR) according to the shape and size of the

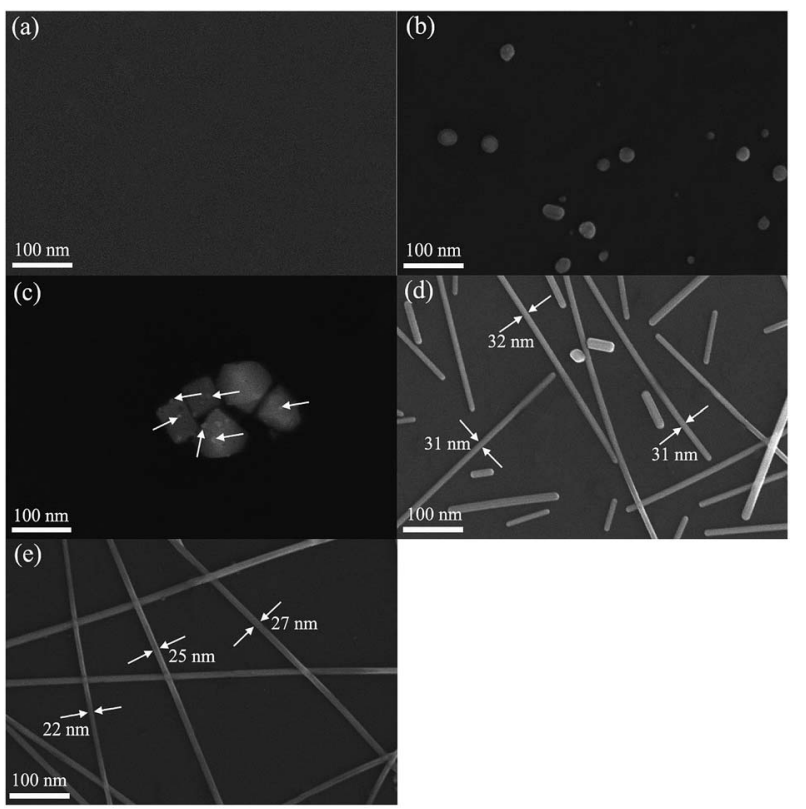

Fig. 7 FE-SEM images of samples at different reaction times: (a) standing at room temperature for $2 \mathrm{~h}$, (b) heating for $5 \mathrm{~min}$, (c) heating for $10 \mathrm{~min}$, (d) heating for $15 \mathrm{~min}$, (e) heating for $20 \mathrm{~min}$. metals. ${ }^{\mathbf{1 6}}$ In essence, the UV-vis spectrum can be used to determine the size and shape of the AgNWs because different frequencies correspond to different SPR bands. ${ }^{17}$ As shown in Fig. 8(a), no apparent absorption peak arose after standing at room temperature for $2 \mathrm{~h}$. The $\mathrm{Ag}^{0}$ nucleis were then formed by heating at $170{ }^{\circ} \mathrm{C}$ for 5 minutes (Fig. 8(b)). As can be seen from Fig. 8(c), there were a distinct peak at $417 \mathrm{~nm}$ and a slightly tilted peak at wavelength of $600 \mathrm{~nm}$, indicating that nanorods formed at this time, but the number was very small and almost invisible by FE-SEM (Fig. 7(c)). From Fig. 8(d), two distinctly different SPR peaks at $354 \mathrm{~nm} 371 \mathrm{~nm}$ could be seen, indicating that AgNWs were generated after $15 \mathrm{~min}$ of reaction at $170{ }^{\circ} \mathrm{C}$. However, the intensity of the SPR peaks at this time was low, implying that only a small amount of AgNWs were produced. As shown in Fig. 8(e), two strong SPR peaks (359 and $368 \mathrm{~nm}$ ) appeared at significantly shorter wavelengths than the SPR peaks in Fig. 8(d), indicating that a large number of finer AgNWs were eventually synthesized.

Based upon the above results and discussions, the possible growth mechanism of AgNWs was proposed, as shown in Fig. 9. In recent years, there were some studies reported that the diameter of AgNWs could be determined by the size of $\mathrm{AgBr}$ seed crystal formed in the prime step of the polyol synthesis. ${ }^{\mathbf{1 8}}$ For the $\mathrm{NaBr}$ additive, the diameter of AgNWs between $30 \mathrm{~nm}$ and $50 \mathrm{~nm}$ were received. ${ }^{19}$ In this paper, however, it can be clearly seen from Fig. 5(a) that the AgNWs with the diameter $<30 \mathrm{~nm}$ even the finest diameter $22 \mathrm{~nm}$ were obtained by the $6 \mathrm{CHZB}$ assistant polyol process. In this process, 6CHZB should play a very important role, which structure is shown in Fig. 9(b). Because the AgNWs grew on the basis of the silver decahedral seeds formed in the early stage, it could be thus inferred that the 6CHZB additive was more favorable for forming small silver decahedral seeds (Fig. 7(c)). Besides, as is known that the interaction of PVP macromolecule with the $\{100\}$ facets is stronger than the $\{111\}$ facets, and this interaction became stronger when the molecular weight of PVP was larger. Also, the bromide ions have been proved to hold an analogous role, adsorbed in the $\{100\}$ facets, consuming to passivate the newly formed $\{100\}$ facets, $\{111\}$ facets at the corner sites would be thereby

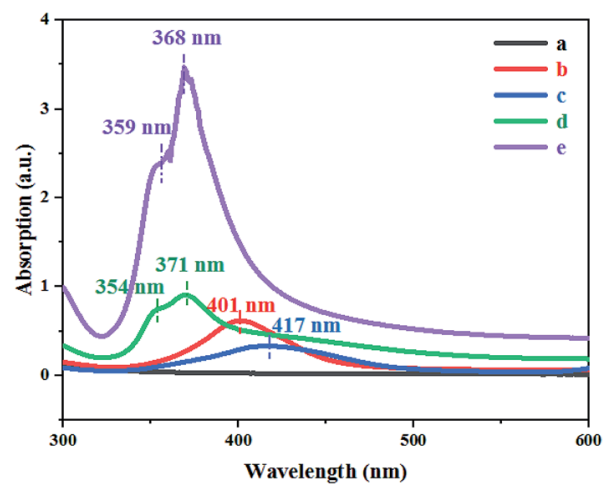

Fig. 8 UV-vis absorption spectra of AgNWs at different reaction times: (a) standing at room temperature for $2 \mathrm{~h}$, (b) heating for $5 \mathrm{~min}$, (c) heating for $10 \mathrm{~min}$, (d) heating for $15 \mathrm{~min}$, (e) heating for $20 \mathrm{~min}$. 


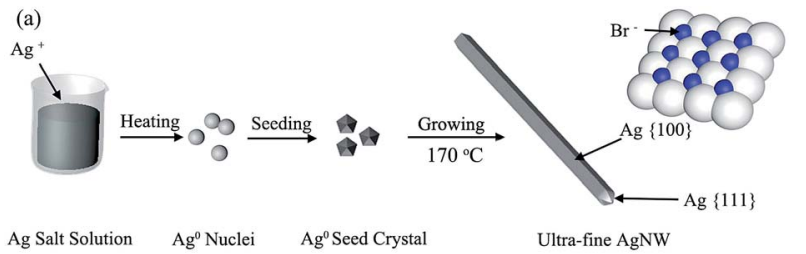

(b) $\mathrm{Br}^{-} \mathrm{Zn}^{+} \overbrace{\mathrm{Cl}}$

Fig. 9 (a) Schematic representation of the proposed growth mechanism of AgNWs, (b) molecular structure of the $6 \mathrm{CHZB}$.

developed and resulted in the formation of ultrafine AgNWs (Fig. 9(a)). ${ }^{19-23}$ On the other hand, the molar concentration of 6CHZB also affected the diameter of AgNWs. A suitable molar concentration increased the likelihood of combination of the photoelectron with interstitial silver, $\mathrm{Ag}^{+}$in the $\mathrm{AgBr}$ seed crystals. Eventually, the latent images formed faster in $\mathrm{AgBr}$ than that in $\mathrm{AgCl}$, partially owing to the different effective mass of electrons $\left(0.215 m_{0}\right.$ vs. $\left.0.302 m_{0}\right)$ and Hall mobility (60 $\mathrm{cm}^{2} \mathrm{~V}^{-1} \mathrm{~s}^{-1}$ vs. $50 \mathrm{~cm}^{2} \mathrm{~V}^{-1} \mathrm{~s}^{-1}$ ) for $\mathrm{AgBr}$ and $\mathrm{AgCl} .^{24}$ This may explain why it took a short time to form ultra-fine AgNWs through 6CHZB additive.

Further, the high-quality AgNWs synthesized by the above method were used to fabricate 2D TCFs by a straightforward bar coating process. Subjectively, as shown in Fig. 10(a), the text below the 2D TCF can be visually observed. In order to verify this phenomenon more scientifically, the transmittance and haze instrument was employed. It can be clearly seen from Fig. 10(d) that the 2D AgNWs film has a high transmittance of $90.3 \%$ and an ultra-low haze of $0.99 \%$ (Fig. 10(c)). In fact, the AgNWs film formed by the ultrafine AgNWs network was nearly transparent due to the intensity of the scattered light is low. In addition, from Fig. 10(b) we can see that the AgNWs film has a low resistance $48 \Omega \mathrm{sq}^{-1}$, suggesting that the film has a good conductivity, which is sufficient to meet the requirements of electronic components.

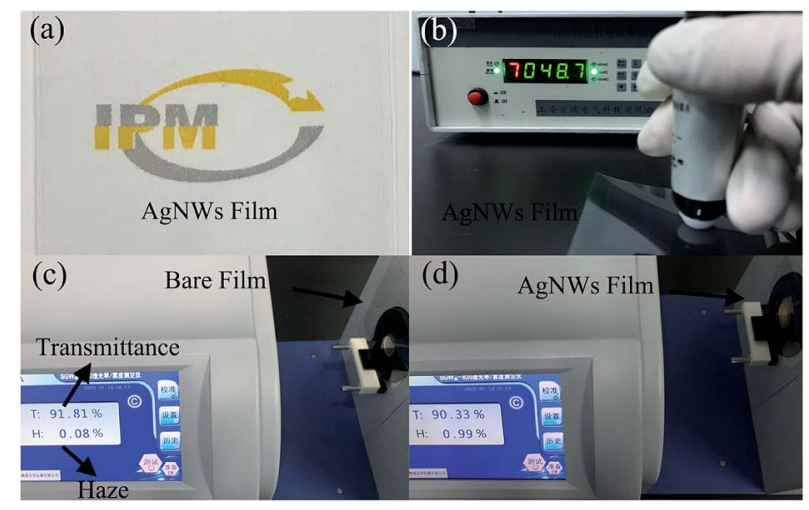

Fig. 10 (a) Optical image of the AgNWs film, (b) sheet resistance measurement of the AgNWs film, (c) the transmittance and haze of the bare PET film, (d) the transmittance and haze of the AgNWs film.

\section{Conclusion}

For the first time, 6CHZB was used as a promoter to successfully synthesize high-quality AgNWs with a diameter of $20 \mathrm{~nm}$ and a length of $40 \mu \mathrm{m}$. The presence of a moderate amount of 6CHZB has been shown to facilitate the formation of smaller silver decahedral seeds and ultimately to form high quality AgNW. More importantly, transparent conductive film fabricated from AgNWs exhibits a high transmittance of $90.3 \%$ and a low haze of $<1.0 \%$ with a low resistance $48 \Omega \mathrm{sq}^{-1}$, which showed great potential to replace the conventional brittle ITO films.

\section{Conflicts of interest}

There are no conflicts to declare.

\section{Acknowledgements}

This work was supported by the National Natural Science Foundation of China (Grant No. 21761016), the Young and Middle-aged Academic and Technical Leaders Reserve Talents Program of Yunnan Province (Grant No. 2017HB060), the Applied Basic Research Foundation of Yunnan Province (Grant No. 2017FB142), the Major R\&D Project of Yunnan Province (Grant No. 2018ZE001), the Research Foundation of Key New Products of Yunnan Province (Grant No. 2016BA007), and the Research Foundation of Institute of Yunnan Province (Grant No. 2016DC033).

\section{Notes and references}

1 K. Ellmer, Nat. Photonics, 2012, 6(12), 9-17.

2 X. Zhang, X. Yan, J. Chen and J. Zhao, Carbon, 2014, 69, 437443.

3 A. T. Khalil, M. Ovais, I. Ullah, M. Ali, Z. K. Shinwari, S. Khamlich and M. Maaza, Nanomedicine, 2017, 12(15), 1767-1789.

4 P. L. Jun, J. L. Jia, L. Lu, F. Ren, W. Hu, J. Li, S. H. Qi and Q. B. Pei, ACS Nano, 2014, 8(12), 12874-12882.

5 T. Aernouts, P. Vanlaeke, W. Geens, J. Poortmans, P. Heremans, S. Borghs, R. Mertens, R. Andriessen and L. Leenders, Thin Solid Films, 2004, 451(452), 22-25.

6 S. Yao and Y. Zhu, Nanoscale, 2014, 6(4), 2345-2352.

7 X. J. Xu, G. T. Fei, X. W. Wang, Z. Jin, W. H. Yu and L. D. Zhang, Mater. Lett., 2007, 61(1), 19-22.

8 J. Xu, J. Hu, C. J. Peng, H. L. Liu and Y. Hu, J. Colloid Interface Sci., 2006, 298(2), 689-693.

9 Y. J. Zhu and X. L. Hu, Mater. Lett., 2004, 58(9), 1517-1519.

10 T. You, S. L. Xu, S. X. Sun and X. Y. Song, Mater. Lett., 2009, 63(11), 920-922.

11 R. R. D. Silva, M. X. Yang, S.-I. Choi, M. F. Chi, M. Luo, C. Zhang, Z. Y. Li, P. H. C. Camargo, S. J. L. Ribeiro and Y. N. Xia, ACS Nano, 2016, 10(8), 7892-7900.

12 E. J. Lee, M. H. Chang, Y. S. Kim and J. Y. Kim, APL Mater., 2013, 105(2), 4211848-4211854. 
13 X. L. Tang, M. Tsuji, P. Jiang, M. Nishio, S. M. Jang and S. H. Yoon, Colloids Surf., A, 2009, 338(1-3), 33-39.

14 B. Wu, A. Heidelberg and J. J. Boland, Nano Lett., 2006, 6(3), 468-472.

15 E. Sumesh, M. S. Bootharaju and T. Pradeep, J. Hazard. Mater., 2011, 189(1-2), 450-457.

16 M. A. Khan, A. Qayyum, I. Ahmed, T. Iqbal, A. A. Khan, R. Waleed, B. Mohuddin and M. Malik, Chin. Phys. Lett., 2016, 33(7), 078102.

17 Y. L. Wang, T. Herricks and Y. N. Xia, Nano Lett., 2003, 3(8), 1163-1166.

18 Q. Zhang, C. H. Moran, X. Xia, M. Rycenga, N. X. Li and Y. N. Xia, Langmuir, 2012, 28(24), 9047-9054.
19 M. Li, M. Jing, Z. Wang, B. Li and X. Q. Shen, J. Nanosci. Nanotechnol., 2015, 15(8), 6088-6093.

20 J. P. Kottmann, O. J. F. Martin, D. R. Smith and S. Schultz, Phys. Rev. B: Condens. Matter Mater. Phys., 2001, 64(23), 235402.

21 Y. G. Sun and Y. N. Xia, Analyst, 2003, 128(6), 686-691.

22 Y. J. Rui, W. L. Zhao, D. W. Zhu, H. Y. Wang, G. L. Song, M. T. Swihart, N. Wan, D. W. Gu, X. B. Tang, Y. Yang and T. Y. Zhang, Nanomaterials, 2018, 161(3), 103390.

23 J. F. Hamilton, Adv. Phys., 1988, 37(4), 359-441.

24 P. Zhang, Y. L. Wei, M. Ou, Z. Z. Huang, S. D. Lin, Y. Y. Tu and J. W. Hu, Mater. Lett., 2018, 213, 23-26. 